\title{
Zwei Fälle ausschließlich submuköser atypischer Karzinoide des supraglottischen Larynx. Welchen Stellenwert hat die schnittbildgebende Diagnostik?
}

Atypische Karzinoide (AK) sind seltene Tumoren des oberen Aerodigestivtraktes und im Larynx überwiegend als supraglottisch lokalisiert beschrieben. Aufgrund der lichtmikroskopischen Ähnlichkeit mit wenig differenzierten oder undifferenzierten Plattenepithelkarzinomen wird vermutet, dass AK des Larynx gelegentlich nicht als solche erkannt und klassifiziert werden. Eine verlässliche Prävalenzrate kann deshalb nicht angegeben werden. AK sind mäßig differenziert und gehören neben den noch weitaus selteneren typischen Karzinoiden (gute Differenzierung) und kleinzelligen Karzinomen (geringe Differenzierung) zu den neuroendokrinen Tumoren epithelialen Ursprungs, zu den sogenannten neuroendokrinen Karzinomen. Die hohe Aggressivität atypischer Karzinoide im Vergleich zu den in über 95\% aller Larynxtumoren vorkommenden Plattenepithelkarzinomen erfordert eine erweiterte Fernmetastasensuche und großzügige Resektionsgrenzen, gegebenenfalls eine radikale Operation, weswegen eine prätherapeutische histopathologische Differenzierung so bedeutsam ist. Da diese nur durch immunhistochemische Zusatzuntersuchungen möglich ist (Dieler et al, Eur Arch Otorhinolaryngol 1995; 252: 229; Ferlito et al, J Laryngol Otol 1998; 112: 827), soll hier anhand von zwei Fällen aufgezeigt werden, inwiefern CT bzw. MRT im Rahmen der Diagnostik wegweisend hilfreich sein können.

\section{Fallbeschreibungen}

1. Fall (51/w): Vor dem Hintergrund eines regelmäßigen Nikotingenusses bemerkte die Patientin seit wenigen Wochen ein zervikales Druckgefühl auf Höhe des Kehlkopfes links sowie eine Heiserkeit. Bei der direkten Laryngoskopie in Narkose fand sich links supraglottisch eine palpatorisch derbe Schleimhautvorwölbung. Sowohl die CT als auch die MRT zeigten eine ausschließlich submuköse, 1,5 cm große homogen KM-aufnehmende Raumforderung (Abb.1 a, b) zwischen Taschenfalte und infrahyoidaler Epiglottis links. Die im Rahmen der Stützendoskopie gezielt durchgeführten transmukösen Biopsien ergaben nach immunhistochemischer Aufarbeitung Anteile eines atypischen Karzinoids (Abb.1 c). Metastasensuspekte zervikale Lymphknoten oder Fernmetastasen waren nicht nachweisbar, so dass ein supraglottisches Larynxmalignom des Stadiums $\mathrm{T}_{2} \mathrm{~N}_{0} \mathrm{M}_{0}$ diagnostiziert wurde. Therapeutisch erfolgte nach funktioneller Neck dissection links und Tracheotomie eine vertikale Hemilaryngektomie links mit Verschluss des Larynxdefekts durch einen Platysma-Faszien-Lappen. Nach Abschluss der primären Wundheilung wurde eine perkutane Strahlentherapie des Larynx sowie des zervikalen bis supraklavikulären Lymphabflussgebietes mit einer Gesamtdosis von $50 \mathrm{~Gy}$ angeschlossen. Der postoperative seit einem halben Jahr andauernde Verlauf gestaltete sich bisher komplikationslos.

2. Fall (34/w): Nach mehrwöchigen Schluckbeschwerden fand sich bei der direkten Laryngoskopie in Narkose eine Verdickung der linken aryepiglottischen Falte mit Übergang auf den Epiglottisrand ohne oberflächliche Tumoranteile. In Zusammenschau mit diesem Befund zeigte die CT eine ausschließlich submukös lokalisierte, $2 \mathrm{~cm}$ große KM-aufnehmende präepiglottische Raumforderung mit Übergang auf die linke aryepiglottische Falte (Abb.2a). Unter der initialen auswärtigen histopathologischen Diagnose eines primären Schmincke-Tumors des supraglottischen Larynx wurde die Patientin zunächst auswärtig einer perkutanen Strahlentherapie mit einer Gesamtdosis von $72 \mathrm{~Gy}$ zugeführt. Aufgrund einer Beschwerdepersistenz erfolgte drei Monate nach Abschluss der Bestrahlung eine erneute Stützendoskopie in Narkose. Neben postradiogenen Veränderungen fand sich eine Verdickung der linken aryepiglottischen Falte bis zur laryngealen Epiglottisfläche (Abb. 2 b). Eine erneute CT zeigte den lokalen Befund unverändert, konnte aber nicht eindeutig zwischen radiogenen Veränderungen und einem Tumorrest differenzieren. Gezielte transmuköse Biopsien ergaben
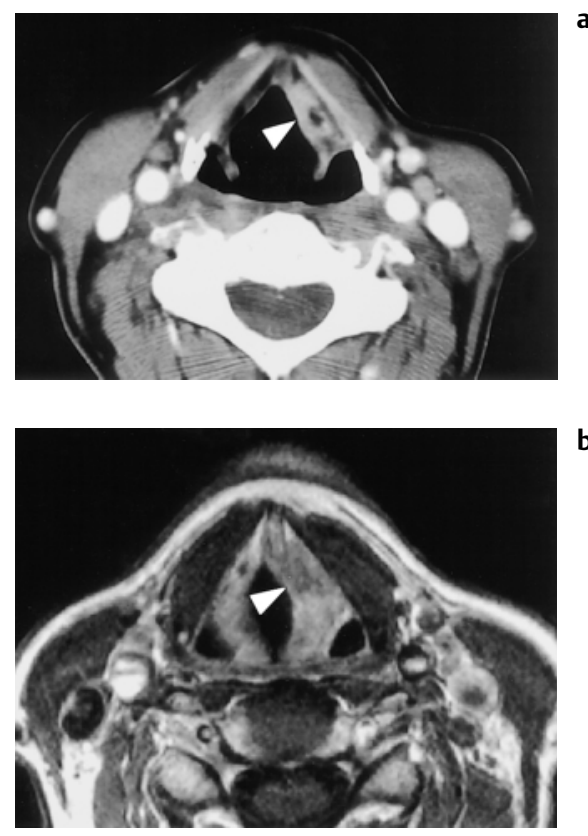

b

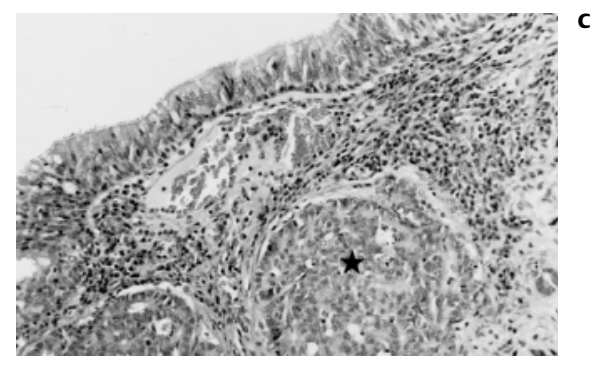

Abb. 1 (a-c) Erster Fall. (a) Die CT zeigt einen links supraglottischen Tumor unter glatt begrenzter leicht vorgewölbter Mukosa (Pfeilspitze). (b) In der $\mathrm{T}_{1}$-gewichteten MRT nach KM-Gabe lässt sich die Mukosa (Pfeilspitze) eindeutig vom submukös gelegenen Tumor abgrenzen. (c) Solides, unter intaktem Oberflächenepithel wachsendes, mäßig differenziertes Karzinom (atypisches Karzinoid) $\left({ }^{*}\right.$ ) mit leicht vergrößerten monomorphen Zellkernen (H.E. × 125). Die Tumorzellen waren negativ für den plattenepithelialen Marker Zytokeratin $5 / 6$ und kräftig positiv für den neuroendokrinen Marker Chromogranin (ohne Abbildung).

den immunhistochemischen Befund eines atypischen Karzinoids (Abb. 2c). Wegen zervikalen Lymphknotenbefalls beidseits wurde nach abgeschlossenem Staging von einem klinischen Tumorstadium $T_{2} \mathrm{~N}_{2 c} \mathrm{M}_{0}$ ausgegangen. Therapeutisch wurde eine transorale laserchirurgische supraglottische Kehlkopfteilresektion, rechtsseitig eine elektive und linksseitig eine modifizierte Neck dissection unter Mitentfernung der V.jugularis interna durchgeführt. Bei der Patientin bildete sich 6 Monate später ein Lokalrezidiv aus, und sie 


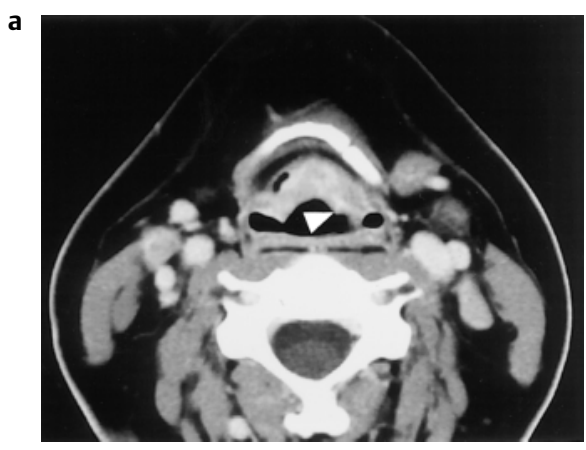

b

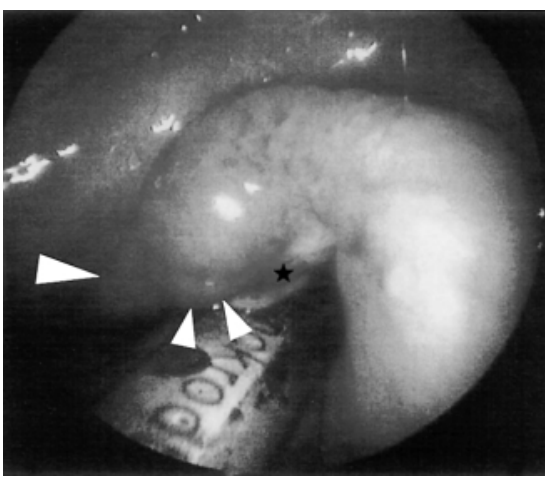

C

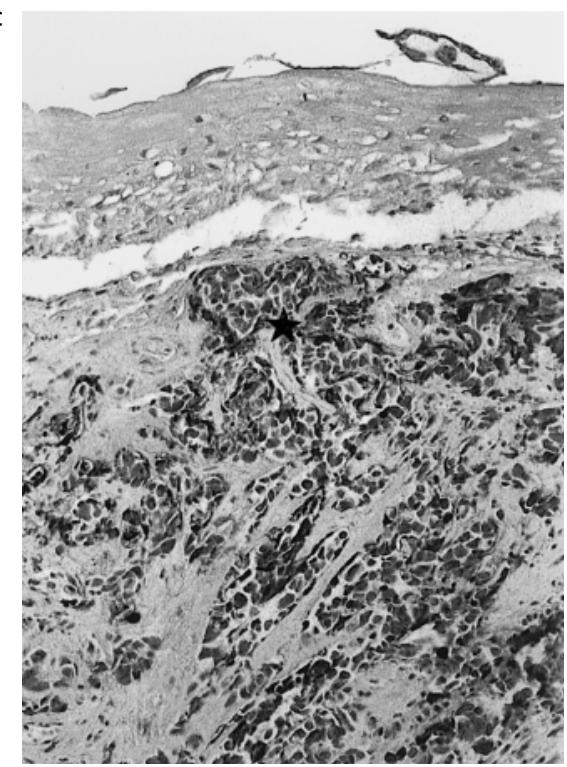

Abb. 2 (a-c) Zweiter Fall. (a) Die CT zeigt einen präepiglottischen Tumor. Am Übergang zwischen laryngealer Epiglottisfläche und linker aryepiglottischer Falte ist eine kleine tumorbedingte Vorwölbung erkennbar (Pfeilspitze). (b) Abgesehen von radiogenen Veränderungen präsentiert sich stützendoskopisch eine derb palpatorische Schleimhautvorwölbung (kleine Pfeilspitzen) am Übergang zwischen laryngealer Epiglottis und aryepiglottischer Falte links (große Pfeilspitze). Erste von mehreren transmukösen Biopsiestellen ( $\left.{ }^{*}\right)$. (c) Mäßig differenziertes, unter intakter Schleimhaut wachsendes neuroendokrines Karzinom $\left({ }^{*}\right)$ mit dichtem hyalinen desmoplastischen Stroma (immunhistochemische Chromogranin-Darstellung, indirekte Peroxidase, $\times 125$ ). verstarb zwei Jahre später bei Vorliegen von multilokulären Fernmetastasen.

\section{Diskussion}

Atypische Karzinoide (AK) des oberen Aerodigestivtrakts stammen wahrscheinlich von Zellvorstufen des seromukösen Drüsenapparates ab. Es ist vor allem das männliche Geschlecht im Alter von 50-70 Jahren betroffen. Ein Nikotinabusus gilt als Risikofaktor. Abgesehen davon, dass die zumeist im supraglottischen Larynx vorkommenden AK üblicherweise submukös wachsen, entspricht ihre lokale Ausbreitungstendenz der von Plattenepithelkarzinomen. Auch die Häufigkeit einer zervikalen Lymphknotenmetastasierung ähnelt mit $43 \%$ der von Plattenepithelkarzinomen. Ihre Aggressivität drückt sich vor allem durch die Tendenz zur Fernmetastasierung in $45 \%$ der Fälle aus. Hier spielen (sub-)kutane Metastasen in $22 \%$ der Fälle vor Lungen-, Leber- und Knochenmetastasen die größte Rolle. Die 5 -Jahresüberlebensrate liegt bei $48 \%$, die 10 -Jahresüberlebensrate bei $30 \%$. Die Symptome Dysphagie, Globusgefühl Heiserkeit oder äußerlich erkennbare Halsschwellung sind unspezifisch. Aufgrund der submukösen Lage können $\mathrm{AK}$ sowohl indirekt als auch direkt laryngoskopisch leichter übersehen werden als die typischerweise oberflächlich wachsenden Plattenepithelkarzinome. Vor diesem Hintergrund kommt der schnittbildgebenden Diagnostik eine große Bedeutung zu: 1. Abgesehen von der guten Beurteilbarkeit der Tiefenausdehnung laryngopharyngealer Tumoren wird der CT bzw. der MRT in Ergänzung zur klinischen Untersuchung ein hoher Nutzen bei der Detektion submuköser Tumoren zugewiesen (Keberle et al. Eur Radiol 1999; 9: 1843). Hierzu sind eine Schichtkippung parallel zur Stimmlippenebene (bzw. zum Zungenbein), eine Kollimation von maximal $3 \mathrm{~mm}$, eine ausreichende Kontrastmittelanflutung und (zusätzliche) Phonationsaufnahmen erforderlich, damit auch kleinere submuköse Tumoren erkannt werden können. Die MRT sollte sowohl $\mathrm{T}_{1}$-gewichtet vor und nach KM-Gabe als auch als $\mathrm{T}_{2-}$ gewichtete TSE-Sequenz durchgeführt werden. Nach KM-Gabe ist eine ergänzende Fettsättigungssequenz sinnvoll. Zur Darstellung des submukösen Wachstums ist die anatomische Auflösung wichtiger als die zeitliche, weswegen schnelle dynamische Gradientenechosequenzen keine Vorteile bringen. Die
Datenakquisition sollte in mindestens zwei Raumebenen erfolgen, die Schichtdicke maximal 4-5 $\mathrm{mm}$ betragen und die Matrix hochauflösend (512er Matrix) sein. 2. Das Ziel dieser Arbeit ist die ausschließlich submuköse Lokalisation der beiden hier veröffentlichten Fälle mit AK als ein für den supraglottischen Larynx auffälliges Merkmal hervorzuheben. Vor dem Hintergrund, dass typische Plattenepithelkarzinome immer oberflächliche Tumoranteile haben, ist das Merkmal einer submukösen Tumorausbreitung bereits zuvor in der Literatur als charakeristisch für das seltene lymphoepitheliale Karzinom, das adenoidzystische Karzinom, Chondrosarkom, Plasmazellgranulom, für vasogene und lipomatöse Tumoren sowie Tumoren des lymphoretikulären Systems beschrieben worden (Becker et al., RadioGraphics 1998; 18: 1189). Entsprechende durch CT bzw. MRT dokumentierte Fälle mit AK sind unseres Wissens noch nicht veröffentlicht, obwohl bereits mehrere Publikationen dieses Merkmal aus histopathologischer Sicht bestätigt haben. Der diagnostische Wert dieses Merkmals wird allerdings dadurch limitiert, dass AK mit zunehmender Größe auch oberflächliche Tumoranteile aufweisen können. Außerdem kann es mit schnittbildgebender Diagnostik in manchen Fällen schwer sein, eindeutig zwischen submukösem und oberflächlichem Tumor zu unterscheiden. Zur Differenzierung ist hier ergänzend die Auskunft durch den Endoskopiker hilfreich, um zur Diagnose eines soliden submukösen Tumors zu gelangen. Dies wiederum sollte den Radiologen dazu veranlassen, die zuvor genannten seltenen Tumoren differenzialdiagnostisch in Erwägung zu ziehen, damit pathologischerseits zusätzlich zur histopathologischen Routine spezielle immunhistochemische Tests durchgeführt werden. Diese gelten als Methode der Wahl, indem neuroendokrine Differenzierungsmerkmale zusätzlich zu epithelialen Charakterisierungen nachgewiesen werden (z. B. Dieler et al.). Die routinemäßig durchgeführte lichtmikroskopisch-histologische Untersuchung alleine erlaubt oft keine eindeutige Differenzierung zwischen wenig differenzierten oder undifferenzierten Plattenepithelkarzinomen, lymphoepithelialen Karzinomen und neuroendokrinen Karzinomen. Vor dem Hintergrund der hohen Aggressivität von AK im Vergleich zu Plattenepithelkarzinomen liegt es nahe, dass der Radiologe bei einer ausschließlich submukösen Tu- 
morlokalisation auf eine für diese Region untypische Histologie eines supraglottischen Larynxtumors zum Beispiel auf ein neuroendokrines Karzinom hinweist. 3. Submuköse Tumoren erfordern transmuköse („tiefe“) Biopsien, die im Rahmen einer direkten Stützendoskopie in Narkose durchgeführt werden. Für eine erfolgreiche Biopsie besteht die Aufgabe schnittbildgebender Verfahren nicht nur in der exakten Abgrenzung der submukösen Tumorausdehnung, sondern auch darin, den Endoskopiker auf die vielversprechendste Biopsiestelle hinzuweisen. Hierzu können koronare Rekonstruktionen aus dem CT-Datensatz hilfreich sein, um die Tumoren speziell in der kraniokaudalen Ausdehnung besser abzugrenzen (Brüning et al, Radiologe 1999; 39: 939). So lässt sich die Genauigkeit tiefer Biopsien steigern und die Zeitdauer bis zur endgültigen Diagnose reduzieren.

Die Diagnose eines AK hat zunächst die Konsequenz eines erweiterten Stagings, wobei neben einer Somatostatinrezeptor-Szintigraphie ein Thorax- und Abdo-
men-CT diejenigen Methoden darstellen, welche suffizient die häufigsten Metastasierungsorgane abdecken. Therapeutisch besteht weitgehend Konsens darin, dass die Resektionsgrenzen großzügig zu wählen sind und dass gegebenenfalls radikal operiert werden sollte. Wie Fall 2 zeigt, reagieren AK (sowie auch typische Karzinoide) nicht ausreichend auf Bestrahlung und auch nicht auf Chemotherapie. Eine funktionelle oder radikale Neck dissection sollte in allen Fällen durchgeführt werden. Fernmetastasen (v.a. schmerzhafte Hautfiliae) sollten lokal exzidiert werden.

Anhand zweier Fälle wird gezeigt, wie schnittbildgebende Verfahren zur Detektion solider submuköser Raumforderungen beitragen, den Verdacht auf das Vorliegen eines atypischen Karzinoids lenken und dem Endoskopiker zu einer erfolgreichen tiefen Biopsie verhelfen können.

M. Keberle, P. Ströbel, R. Dieler, Würzburg 